\title{
RESEARCH HIGHLIGHT Guiding a mutator in antibody diversification
}

\author{
Ming $\operatorname{Tian}^{1}$ and Frederick W. Alt ${ }^{1}$ \\ Cell Research (2018) 28:963-964; https://doi.org/10.1038/s41422-018-0085-8
}

\begin{abstract}
Activation Induced Cytidine Deaminase is a mutator that is essential for antibody diversification. A new study, recently reported in Cell Research, provides insights into the mechanism that guides the mutator to physiological targets.
\end{abstract}

The immune system produces diverse antibodies to counter infections. Three mechanisms contribute to antibody diversity (Fig. 1). ${ }^{1}$ During B cell maturation, $\mathrm{V}(\mathrm{D}) \mathrm{J}$ recombination creates a large repertoire of antigen-binding sites in immunoglobulin (Ig) variable regions. Naïve $B$ cells express the primary antigen-binding sites in the context of B cell receptors. Antigenic challenge triggers $B$ cell activation. In this process, class switch recombination (CSR) changes the constant region of $\lg$ heavy chain $(\operatorname{lgH})$, which defines antibody class. All naïve $B$ cells express $\lg M$ and $\lg D$, whereas activated B cells express lgG, IgA or IgE. As different classes of antibody are specialized for distinct immune functions, class switching broadens the scope of immune response. In parallel with CSR, somatic hypermutation (SHM) modifies antibody variable regions to improve antigen-binding affinity.

CSR takes place between switch regions that precede constant regions at the $\mathrm{IgH}$ locus (Fig. 1). SHM introduces primarily point mutations into the variable region exons of both lg heavy and light chains. Despite their apparent differences, CSR and SHM share similar mechanisms. ${ }^{2,3}$ At the core of both processes is Activation Induced Cytidine Deaminase (AID). AID initiates CSR by deaminating cytidines in switch regions. The deamination leaves uracil in DNA. Uracil DNA glycosylase excises the uracil to form abasic sites. Apurinic endonuclease cleaves the abasic sites to break switch regions. Non-Homologous End Joining machinery ligates two broken switch regions to form the CSR product. During SHM, AID deaminates cytidines in Ig variable region exons. Subsequent processing of uracil by DNA replication and various repair pathways mutate the variable region.

The mutagenic activity of AID is largely confined to switch regions and Ig variable regions. The restriction is not absolute, and AID acts on certain non-lg loci at low levels. Despite the leakiness, the targeting mechanism minimizes deleterious mutations in $B$ cells during an immune response. Oncogenic transformation of $B$ lymphocyte is relatively rare in relation to innumerable $B$ cell activation events throughout the lifespan of an individual. Understanding the AID targeting mechanism has been a major goal of the field.

AID preferentially deaminates cytidines in the context of WRCY or the complementary RGYW motif. Such a prevalent motif cannot be sufficient to unequivocally mark AID targets. Although AID is relatively nondiscriminatory with respect to substrates at the primary sequence level, the enzyme acts strictly on single-stranded DNA. As transcription transiently unwinds DNA template, the process is a major source of single-stranded DNA in genome. Consistent with this link, both CSR and SHM require transcription.
Since AID does not act on all transcribed regions, transcription at AID targets may be special in some way. Indeed, AID off-targets in non-lg loci tend to be convergently transcribed. ${ }^{4,5}$ How convergent transcription attracts AID to off-targets, and whether or not it is involved in on-target activity at $\mathrm{lg}$ loci has not been fully elucidated. The study by Chen and colleagues, which is reported recently in Cell Research, ${ }^{6}$ shed some light on this question.

Chen and colleagues investigated the AID targeting mechanism by searching for factors that interact with AID and guide AID to genomic targets. The approach is a well-trodden path and, over the years, has yielded a bewilderingly large collection of AIDinteracting proteins. However, the role of many of these putative co-factors remains to be rigorously validated by functional assays in vivo. In this crucial respect, the AID-interacting protein identified by Chen and colleagues, ROD1/PTBP3, ${ }^{7}$ stands out from the crowd. To test the function of ROD1, the authors knocked out its gene in mice. Remarkably, ROD1 deficiency led to severe reductions in both CSR and SHM. The phenotype is not an indirect consequence of defects in related processes. In this regard, ROD1knockout mice contain normal B cell populations in different developmental stages, including germinal center B cells, where CSR and SHM take place. In response to $B$ cell activation signals in vitro, the ROD1-deficient B cells proliferate, upregulate AID expression, and transcribe various switch regions at comparable levels as wild-type B cells. In light of the direct interaction between AID and ROD1, the most plausible interpretation of the phenotype is that loss of ROD1 directly impairs AID function. ROD1 protein is a family member of RNA-binding proteins that exhibit high affinity for polypyrimidine tracts. ${ }^{8}$ The protein consists of a tandem array of four RRM-type RNA binding domains. Based on a genome-wide survey, ROD1 preferentially associates with transcripts that originate from bi-directionally transcribed loci. In line with the association between AID and ROD1, AID exhibits similar RNA binding profiles to ROD1, and the interaction is ROD1-dependent. Based on these observations, the authors proposed that ROD1 binds nascent transcripts at bi-directionally transcribed loci and recruits AID to these targets.

The model raises several questions. First, like other polypyrimidine tract-binding proteins, ROD1 preferentially interacts with pyrimidine-rich motifs in RNA. Paradoxically, transcription of one of the most prominent AID targets, switch region, yields predominantly G-rich transcripts. Transcripts from lg variable regions are not notable for high pyrimidine content either. How could ROD1 recognize transcripts from these bona fide AID targets? Second, to guide AID to DNA, ROD1 must bind to nascent transcript that is still attached to template. Interaction with free transcript is counterproductive, as ROD1 would divert AID away from its genomic target. How could ROD1 differentiate a nascent transcript from the swarm of free transcripts with the same

'Boston Children's Hospital, Harvard Medical School, HHMI, Boston, MA 02115, USA

Correspondence: Ming Tian (Ming_Tian@hms.harvard.edu)

Published online: 31 August 2018 

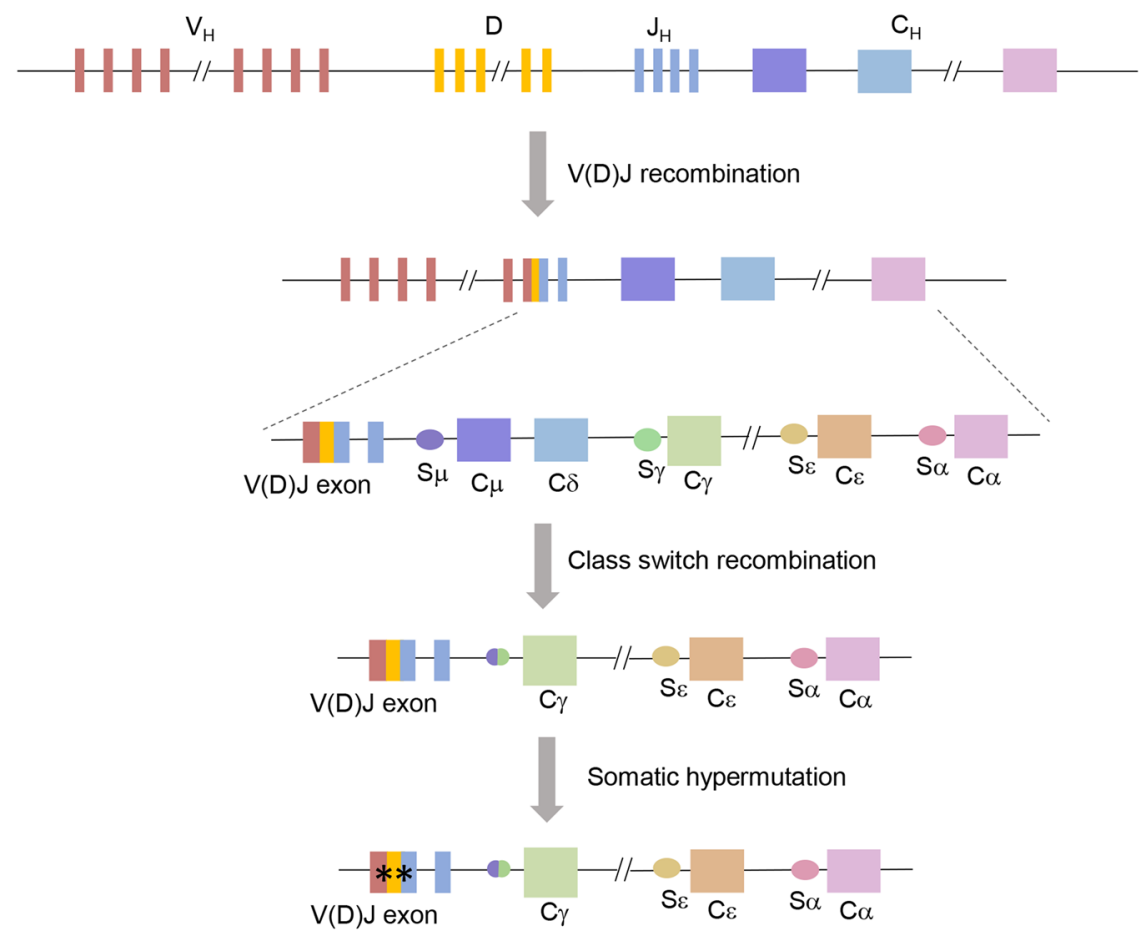

Fig. 1 Illustration of three pathways of antibody diversification. The diagram is for illustration purpose only and is not drawn to scale. The germline $\mathrm{IgH}$ locus is shown at the top. In B cell progenitors, $V(D) J$ recombination joins various combinations of $V_{H}$, $D$, and $J_{H}$ segments together to form the complete $V(D) J$ exon, which encodes the lg variable region. In naïve $B$ cells, $C \mu$ and $C \delta$ are expressed in association with the assembled $V(D) J$ exon to form $\operatorname{lgM}$ and $\lg D$, respectively. In response to antigenic challenge and $B$ cell activation, class switch recombination occurs between switch region $(S \mu)$ in front of $C \mu$ and one of the downstream switch regions, for instance, $S \gamma$ in the diagram. As a result, the downstream constant region (e.g., $C \gamma$ in the diagram) replaces $C \mu$ and $C \delta$, leading to the expression of a new class of antibody with distinct effector functions. Additionally, somatic hypermutation introduces high levels of mutations in the $V(D) J$ exon. The microenvironment of germinal center selects for mutations that improve antigen-binding affinity

sequence? Bi-directional transcription is unlikely to be a specific mark for ROD1 or AID targets either, as divergent transcription near promoters is a common trait of many loci. Instead, convergent transcription provides a more definitive correlation with AID non-lg off-targets, although the association remains to be established for the two main AID targets: switch regions and Ig variable regions. Thus, additional work is needed to determine whether and how ROD1 targets convergently transcribed regions and, more importantly, whether the mechanism applies to the physiological AID targets at the lg loci.

\section{REFERENCES}

1. Alt, F. W., Zhang, Y., Meng, F. L., Guo, C. \& Schwer, B. Cell 152, 417-429 (2013).

2. Methot, S. P. \& Di Noia, J. M. Adv. Immunol. 133, 37-87 (2017).

3. Hwang, J. K., Alt, F. W. \& Yeap, L. S. Microbiol. Spectr. 3, MDNA3-0037-2014 (2015).

4. Meng, F. L. et al. Cell 159, 1538-1548 (2014).

5. Qian, J. et al. Cell 159, 1524-1537 (2014).

6. Chen, J. et al. Cell Res. https://doi.org/10.1038/s41422-018-0076-9 (2018).

7. Yamamoto, H., Tsukahara, K., Kanaoka, Y., Jinno, S. \& Okayama, H. Mol. Cell. Biol. 19, 3829-3841 (1999).

8. Garcia-Blanco, M. A., Jamison, S. F. \& Sharp, P. A. Genes Dev. 3, 1874-1886 (1989). 